\title{
Three novel serum biomarkers, miR-1, miR-133a, and miR-206 for Limb-girdle muscular dystrophy, Facioscapulohumeral muscular dystrophy, and Becker muscular dystrophy
}

\author{
Yasunari Matsuzaka $\cdot$ Soichiro Kishi $\cdot$ \\ Yoshitsugu Aoki · Hirofumi Komaki • \\ Yasushi Oya $\cdot$ Shin-ichi Takeda $\cdot$ Kazuo Hashido
}

Received: 15 April 2014/ Accepted: 12 August 2014/Published online: 24 August 2014

(C) The Japanese Society for Hygiene 2014

\begin{abstract}
Objectives Muscular dystrophies are a clinically and genetically heterogeneous group of inherited myogenic disorders. In clinical tests for these diseases, creatine kinase (CK) is generally used as diagnostic blood-based biomarker. However, because CK levels can be altered by various other factors, such as vigorous exercise, etc., false positive is observed. Therefore, three microRNAs (miRNAs), miR-1, miR-133a, and miR-206, were previously reported as alternative biomarkers for duchenne muscular dystrophy (DMD). However, no alternative biomarkers have been established for the other muscular dystrophies. Methods We, therefore, evaluated whether these miR-1, miR-133a, and miR-206 can be used as powerful biomarkers using the serum from muscular dystrophy patients including DMD, myotonic dystrophy 1 (DM1), limb-girdle muscular dystrophy (LGMD), facioscapulohumeral muscular dystrophy (FSHD), becker muscular dystrophy
\end{abstract}

Y. Matsuzaka, S. Kishi contributed equally to this work.

Electronic supplementary material The online version of this article (doi:10.1007/s12199-014-0405-7) contains supplementary material, which is available to authorized users.

Y. Matsuzaka · S. Kishi · K. Hashido ( $₫)$

Administrative Section of Radiation Protection, National

Institute of Neuroscience, Tokyo, Japan

e-mail: hashido@ncnp.go.jp

Present Address:

S. Kishi

Department of Pathology, Institute for Developmental Research,

Kasugai, Aichi 480-0392, Japan

Y. Aoki $\cdot$ S. Takeda

Department of Molecular Therapy, National Institute of

Neuroscience, Tokyo, Japan
(BMD), and distal myopathy with rimmed vacuoles (DMRV) by qualitative polymerase chain reaction (PCR) amplification assay.

Results Statistical analysis indicated that all these miRNA levels in serum represented no significant differences between all muscle disorders examined in this study and controls by Bonferroni correction. However, some of these indicated significant differences without correction for testing multiple diseases $(P<0.05)$. The median values of miR-1 levels in the serum of patients with LGMD, FSHD, and BMD were approximately 5.5, 3.3 and 1.7 compared to that in controls, 0.68 , respectively. Similarly, those of miR133a and miR-206 levels in the serum of BMD patients were about 2.5 and 2.1 compared to those in controls, 1.03 and 1.32 , respectively.

Conclusions Taken together, our data demonstrate that levels of miR-1, miR-133a, and miR-206 in serum of BMD and miR-1 in sera of LGMD and FSHD patients showed no significant differences compared with those of controls by Bonferroni correction. However, the results might need increase in sample sizes to evaluate these three miRNAs as variable biomarkers.

Present Address:

Y. Aoki

Department of Physiology, Anatomy and Genetics, University of Oxford, South Parks Road, Oxford OX1 3QX, UK

\section{H. Komaki}

Department of Child Neurology, National Center of Neurology and Psychiatry (NCNP), Kodaira, Tokyo, Japan

Y. Oya

Department of Neurology, National Center of Neurology and Psychiatry (NCNP), Kodaira, Tokyo, Japan 
Keywords microRNAs - Biomarker · Limb-girdle muscular dystrophy $\cdot$ Facioscapulohumeral muscular dystrophy $\cdot$ Becker muscular dystrophy

\section{Introduction}

miRNAs are approximately 19-23 nucleotides' long single-stranded non-coding RNAs, and the function is posttranscriptional regulation of target messenger RNAs (mRNAs) [1] dysregulation of miRNAs expression in skeletal muscle and myocardium is associated with muscle disorders [2, 3]. Interestingly, despite the high RNase activity within the circulating blood, a high concentration of these remarkably stable miRNAs has been found in various body fluids, including serum and plasma, as microvesicle-encapsulated [4] or RNA-binding proteinassociated forms [5].

Muscular dystrophies are classified in accordance with their clinical and pathological features [6, 7]. Among them, DMD (OMIM310200) and BMD (OMIM 300376) are caused by various mutations in the dystrophin gene on the $\mathrm{X}$ chromosome, at Xp21.2 [8], and exhibit estimated prevalences of approximately one per 3,500 in DMD and 3-6 per 100,000 in BMD [9]. DM1 (OMIM160900), also known as Steinert's disease, represents an estimated prevalence of 5.5 per 100,000 Japanese [10], and is caused by expansion of a CTG repeat in the $3^{\prime}$ UTR of the DMPK (dystrophia myotonica-protein kinase) gene [11]. LGMD is caused by a total of twenty-two autosomal dominant or recessive causative gene mutations [12], and has an incidence of about one per 20,000 individuals. FSHD (OMIM158900) is caused by a loss of the D4Z4 microsatellite locus on chromosome 4 [13] with a prevalence of approximately one per 20,000 Japanese [6]. DMRV (OMIM605820), also called Nonaka myopathy, hereditary inclusion body myopathy (hiBM), and quadriceps sparing myopathy, is an autosomal recessive vacuolar myopathy of the distal muscles of the tibialis anterior, caused by mutations in the UDP-N-acetylglucosamine 2-epimerase/ $\mathrm{N}$-acetylmannosamine kinase (GNE) gene [14]. The prevalence of DMRV is approximately $300-400$ patients within the Japanese population.

Serum CK values are commonly used as clinical bloodbased biomarkers for these muscular dystrophies. However, there are some problems in using serum $\mathrm{CK}$ values for diagnostic evaluation of these disorders, i.e. CK levels are increased by vigorous exercise [15], decreased renal function due to aging, gender-dependent differences in skeletal muscle mass, pregnancy, and alcohol intake, and does not parallel motor ability in DMD [16, 17]. We, therefore, previously reported that three miRNAs, miR-1,
miR-133a, and miR-206, in the serum were used as novel biomarkers in the dystrophin-deficient muscular dystrophy mouse models, as well as the canine X-linked muscular dystrophy in Japan dog (CXMDj) [18]. Furthermore, another group reported that an increase in miR-1, miR133a, and miR-206 levels in the serum of DMD children patients was correlated with motor ability [17]. In other muscle diseases, however, stable and valuable biomarkers as an alternative to CK have not been established to date.

In this study, we measured and evaluated these three miRNAs, miR-1, miR-133a, and miR-206, in serum as possible stable and powerful biomarkers for DM1, LGMD, LGMAD2B, FSHD, BMD, and DMRV.

\section{Materials and methods}

\section{Patients}

A total of forty-eight unrelated Japanese patients with DMD, DM1, LGMD, LGMD2B, FSHD, BMD, and DMRV and each of the five age-matched controls were enrolled in this study (Table 1). DMD patients were divided into two groups by age (average ages and age ranges were 10.2 and 28.7 years of age, 5-18 and 27-31 years of age, respectively). LGMD patients were divided into two groups by whether they contained LGMD2B or not. Informed consent was obtained from the cases and controls by explaining the details of this study prior to collection of peripheral blood. The Research Ethics Committee for National Institute of Neuroscience, National Center of Neurology and Psychiatry approved the present study and all participants provided written informed consent.

\section{Animals}

All animals used in this study were housed in the National Center of Neurology and Psychiatry and treated in accordance with the guidelines provided by the Ethics Committee for the Treatment of Laboratory Animals of National Institute of Neuroscience, or the Ethics Committee for the Treatment of Laboratory Middle-sized Animals of National Institute of Neuroscience, which has adopted the three fundamental principles of replacement, reduction, and refinement.

RNA extraction and quantification of miRNA

Total RNA was extracted from $50 \mu \mathrm{l}$ of serum using the mirVana miRNA isolation kit (Ambion, Austin, TX, USA) according to the manufacturer's protocol and $50 \mu \mathrm{l}$ of RNA eluate. Five $\mu l$ of the RNA elute was reverse transcribed 
Table 1 Muscle dystrophy patients

$y . a$ years of age

\begin{tabular}{lllllcc}
\hline Disease & $\begin{array}{l}\text { Sample } \\
\text { no. }\end{array}$ & $\begin{array}{l}\text { Average of } \\
\text { age } \pm \text { SD (y.a.) }\end{array}$ & $\begin{array}{l}\text { Sex (male/ } \\
\text { female) }\end{array}$ & $\begin{array}{l}\text { Sporadic/ } \\
\text { Familial }\end{array}$ & $\begin{array}{l}\text { Average of } \\
\text { onset } \pm \text { SD (y.a.) }\end{array}$ & $\begin{array}{l}\text { Serum CK (U/ } \\
\text { ml) }\end{array}$ \\
\hline DMD & 5 & $10.4 \pm 4.2$ & $5 / 0$ & $3 / 2$ & $3.8 \pm 1.1$ & $9,101 \pm 6,282$ \\
DMD & 7 & $28.7 \pm 1.5$ & $7 / 0$ & $7 / 0$ & $3.8 \pm 0.7$ & $220 \pm 126$ \\
DM1 & 8 & $59.4 \pm 17.4$ & $5 / 3$ & $4 / 4$ & $18.4 \pm 16.9$ & $121 \pm 66$ \\
LGMD & 7 & $50.0 \pm 19.8$ & $5 / 2$ & $5 / 2$ & $15.5 \pm 13.9$ & $633 \pm 661$ \\
LGMAD2B & 4 & $51.0 \pm 18.2$ & $3 / 1$ & $4 / 0$ & $23.0 \pm 5.7$ & $2,713 \pm 2,722$ \\
FSHD & 8 & $52.3 \pm 17.3$ & $4 / 4$ & $4 / 4$ & $14.4 \pm 10.8$ & $162 \pm 155$ \\
BMD & 4 & $52.0 \pm 12.9$ & $4 / 0$ & $4 / 0$ & $14 \pm 14.6$ & $687 \pm 562$ \\
DMRV & 5 & $39.2 \pm 7.3$ & $0 / 4$ & $4 / 1$ & $22.2 \pm 6.2$ & $206 \pm 177$ \\
\hline
\end{tabular}

using the TaqMan miRNA Reverse Transcription kit (ABI, Foster City, CA, USA) and miRNA-specific stem-loop primers (part of TaqMan miRNA assay kit: Applied Biosystems) as previously reported [18]. For exosome and exosome-depleted supernatant, 5-fold diluted solutions of the RNA elute were used with distilled water. The expression levels of miRNA were quantified by real-time PCR using individual miRNA-specific primers (part of TaqMan miRNA assay kit: Applied Biosystems) with 7900HT Fast Real-Time PCR System (Applied Biosystems) according to the manufacture's protocol. Each samples were performed real-time PCR as triplicade. Each miRNA expression was represented relative to the expression of miR-16 used as an internal control. Data analysis was performed by SDS 2.1 real-time PCR data analysis software (Applied Biosystems). Expression data were given as median values obtained from three samples in conjunction with standard deviation. Statistical comparisons were performed by Mann-Whitney $U$ test. Bonferroni correction was used to resolve a problem of multiple testing.

\section{Creatine kinase activity}

Serum creatine kinase (CK) levels were measured with the Fuji Dri-chem system (Fuji Film Medical Co. Ltd, Tokyo, Japan) according to the manufacture's protocol. Ten $\mathrm{ml}$ of serum was deposited on a Fuji Dri-chem slide and incubated at $37{ }^{\circ} \mathrm{C}$. The increase in absorbance by the generated dye was measured for $5 \mathrm{~min}$ at $540 \mathrm{~nm}$ spectrophotometrically, and the activity was calculated according to the installed formula. Data were expressed as units per liter (U/1).

\section{Exosome purification}

Serum was harvested from the peripheral blood of DMD patients in tubes by centrifugation at $3,000 \times g$ for $15 \mathrm{~min}$. Isolation of exosome from serum was performed by Exo Quick Exosome Precipitation Solution (System
Biosciences, CA) according to the protocol provided by the manufacturer. Briefly, $63 \mu \mathrm{l}$ of the Exo Quick Exosome Precipitation Solution was added to $250 \mu \mathrm{l}$ of serum. The mixture was vortexed for $15 \mathrm{~s}$ and then incubated at $4{ }^{\circ} \mathrm{C}$ for $30 \mathrm{~min}$. After centrifugation at $1,500 \times \mathrm{g}$ for $30 \mathrm{~min}$ at room temperature, the supernatant was discarded. Again, the centrifugation and aspiration were repeated. The pellet including exosomes was resuspended in $1 \times$ phosphatebuffered saline (PBS).

CTX-induced skeletal muscle regeneration of mice

C57B1/10SnSlc mice were obtained from Clea Japan Inc., and used at 7-8 weeks age. Hair from the bilateral hind limbs of diethyl ether-anesthetized animals was removed with a depilatory cream before the induction of injury. The tibialis anterior (TA) muscle of mice was injured by injection of $100 \mu \mathrm{l}$ of PBS or cardiotoxin (CTX, $10 \mu \mathrm{M})$ (Naja mossambica mossambica, Sigma-Aldrich), a snake venom that selectively injures myofibers by disturbing calcium homeostasis at the neuromuscular junctions, followed by necrosis of muscle fibers [19]. The concentration of cardiotoxin ensures minimal damage to satellite cells and also to the nerves and blood vessels of the original muscles [20]. After 1, 3, and 5 days, whole body blood was collected from the abdominal aorta under anesthesia, and allowed to stand for about $30 \mathrm{~min}$ at room temperature before centrifugation at $1,200 \times g$ for $10 \mathrm{~min}$ at room temperature. The supernatant was used as serum to isolate miRNAs.

\section{Results}

miRNA levels in the serum of patients of various muscle diseases

To assess the validity of miRNAs as alternative serum biomarkers to $\mathrm{CK}$ for various muscle diseases, we analyzed the expression levels of three miRNAs, miR-1, miR-133a, 

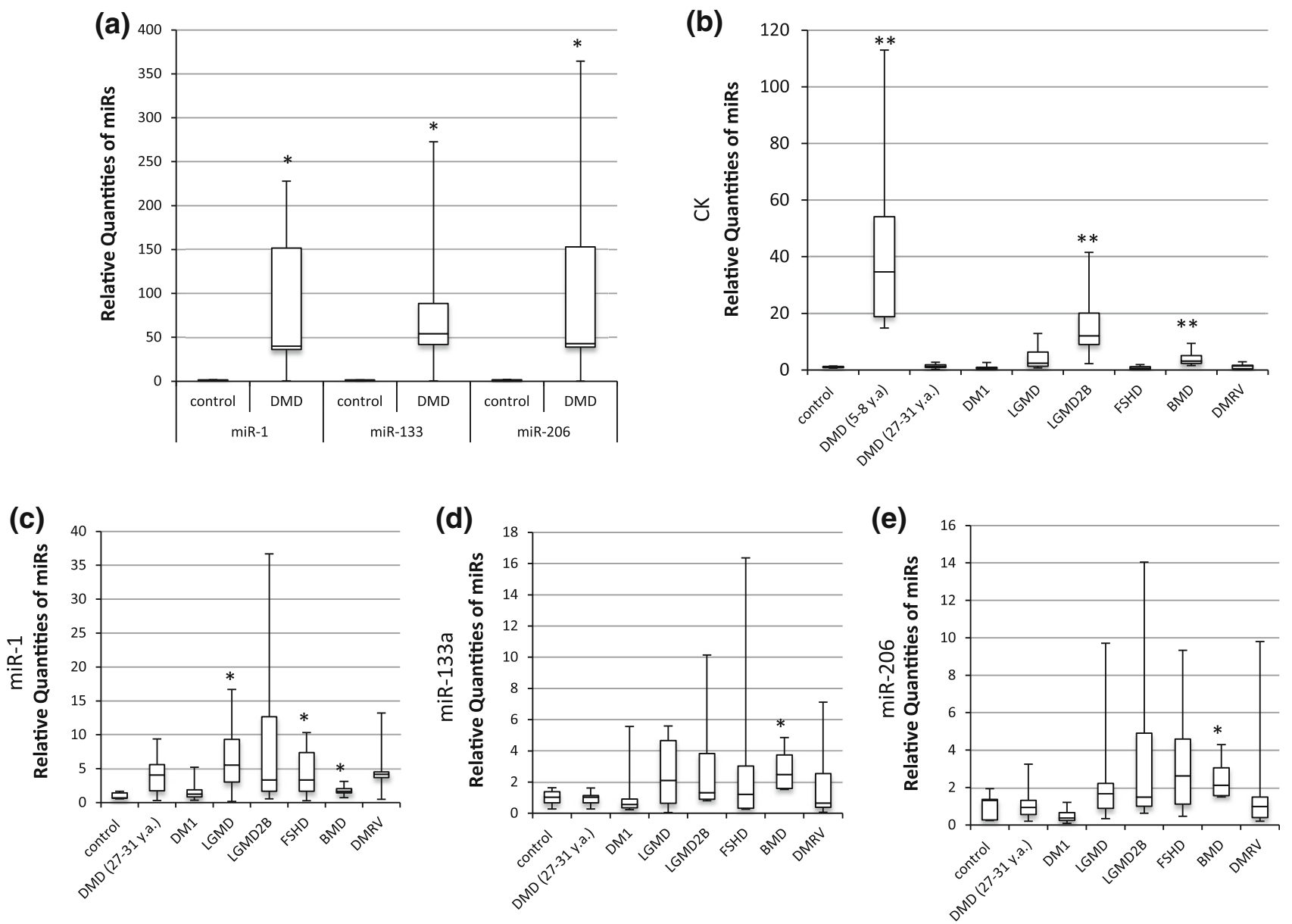

Fig. 1 Evaluation of miRNA levels in various muscular dystrophies indicated by box plot. a Expression levels of miR-1, miR-133a, and miR-206 in the serum of DMD (5-18 years of age) versus control children, evaluated by RT-pPCR. b CK activities in various muscular

and miR-206, in DMD (5-18 and 27-31 years of age), DM1, LGMD, LGMD2B, FSHD, BMD, and DMRV patients and healthy controls. Although all these miRNA levels in sera of all muscle diseases tested in this study represented no significant differences with controls by Bonferroni correction, associations of these miRNA levels with some disorders of them were observed without multiple corrections. As previous reported, qRT-PCR showed that the median values of levels of all three miRNAs, miR1, miR-133a, and miR-206, in the serum of DMD (5-18 years of age) were approximately $39.8,54.0$, and 43.0 compared with those of controls, $0.68,1.03$, and 1.32 , respectively $(P<0.05$, Fig. 1a). The median value of CK activity in the serum of DMD (5-18 years of age) was significantly increased compared with controls (cases verses controls; 34.6 verses $0.89, P<0.01$, Fig. 1b). Although LGMD2B and BMD patients also indicated high median value of $\mathrm{CK}$ activities compared with controls (LGMD2B, BMD verses controls; 12.1, 3.1 verses $0.89, P<0.05$, dystrophies. Expression levels of $\mathbf{c}$ miR-1, d miR-133a, and e miR206 were examined using serum from patients with the indicated muscular dystrophies. Each bar represents mean $\pm \mathrm{SD} . * P<0.05$ versus control by Mann-Whitney $U$ test

Fig. 1b). On the other hand, each median value of miR-1 levels in the serum of LGMD, FSHD and BMD patients was significantly increased compared with controls (LGMD, FSHD and BMD verses controls; 5.5, 3.3, and 1.7 verses $0.68, P<0.05$, Fig. 1c). As for miR-133a and miR206, BMD patients presented significant increases in the median value of expression levels in the serum compared to controls (cases verses controls for miR-133a and miR206; 2.49 verses 1.03 , and 2.13 verses $1.32, P<0.05$, Fig. 1d, e). DM1 and DMRV showed no significant differences with controls for the three miRNAs. Next, we evaluated the three miRNAs as available biomarkers by receiver operating characteristics (ROC) analysis. These results indicated that area under the curve (AUC) in miR-1 displayed $0.83,0.88$, and 0.90 for LGMD, FSHD, and BMD, respectively, in spite of values below 0.8 for DMD (5-18 years of age), DM1, LGMD2B, DMRV (Supplemrntary Fig. 1a). Similarly, the AUC in miR-133a and miR-206 was 0.90 and 0.90 for BMD (Supplementary 

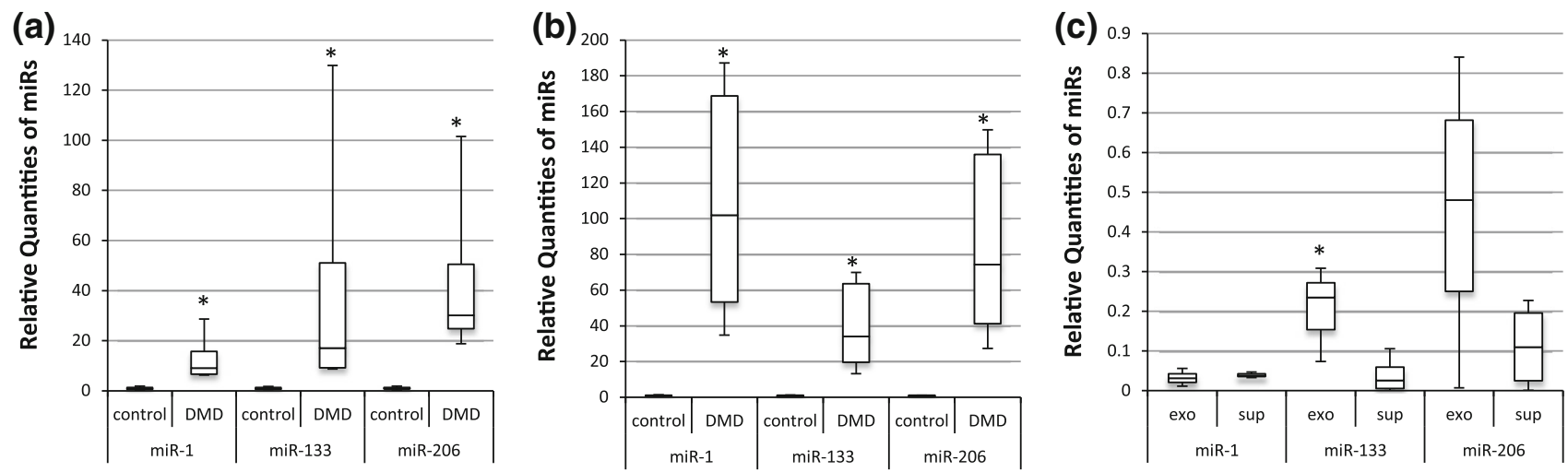

Fig. 2 Expression levels of miR-1, miR-133a, and miR-206 represented by box plot in (a) exosomes or (b) exosome-depleted supernatants extracted from the serum of DMD patients and controls, and analyzed by RT-qPCR. Relative expression of miR-1, miR-133a, and miR-206 is displayed as the difference in the threshold cycle

Fig. 1b, c). miR-1 in LGMD and FSHD, and miR-1, miR133a, and miR-206 in BMD may be useful novel biomarkers.

miRNAs expressions in exosome from the serum of DMD patients

To determine whether the up-regulation of miR-1, miR133a, and miR-206 levels in serum of DMD patients resulted from inclusion of these miRNAs within exosome, we separated serums from patients and controls into exosome and exosome-depleted supernatant by Exo Quick Exosome Precipitation Solution. RNAs extracted from both sources were compared for the levels of the three miRNAs levels by RT-qPCR. The levels of the three miRNAs of serum of DMD patients in both fractions of exosome and exosome-depleted supernatant represented no statistically significant differences with those of controls by Bonferroni correction, but significant differences in these miRNA levels in serum between DMD patients and controls showed without multiple corrections. The median value of levels of each miR-1, miR-133a, and miR-206 in the RNAs within the exosome extracted from the DMD patients showed higher levels, 9.1, 17.0, and 30.1, compared with that of controls, 0.82, 0.77, and 0.99, respectively $(P<0.05$, Fig. 2a). Furthermore, the median value of these miR-1, miR-133a, and miR-206 levels for RNAs from the exosome-depleted supernatant of DMD patients, 101.9, 34.1, and 74.2, exhibited high levels compared with that of controls, $1.0,0.39$, and 0.42 , respectively $(P<0.05$, Fig. 2b). To evaluate whether the majority of these miRNAs in the serum of DMD patients are concentrated in exosome or freely circulating in blood stream, the amount number between miRNA from exosomes or the exosome-depleted supernatant extracted from the serum of DMD patients (c) in five times dilution. Each bar represents mean \pm SD. ${ }^{*} P<0.05$ versus control by Mann-Whitney $U$ test

of the three miRNAs extracted from exosome and exosome-depleted supernatants was measured by RT-qPCR. All these miRNA levels exhibited no significant differences between exosome and exosome-depleted supernatants by Bonferroni correction. However, the levels of miR-1, miR-133a, and miR-206 in both fractions of exosome and exosome-depleted supernatant from the serum of DMD patients are remarkably increased compared with those in controls. The content of miR-133a within exosome is significantly higher than in the exosome-depleted supernatant without multiple corrections (Fig. 2c, $P<0.05)$.

miR-1, miR-133a, and miR-206 levels in mouse serum are up-regulated upon skeletal muscle regeneration

Next, to assess whether the three miRNA levels are affected in skeletal muscle regeneration in vivo, we induced skeletal muscle injury by injecting CTX into the TA of mice, and analyzed the expression of the three miRNAs by RT-qPCR at 1,3 , and 5 days after injection. The levels of miR-1, miR-133a, and miR-206 were increased dramatically by about 15-, 55-, and 15-fold in the serum of CTX-injured mice on day 1 compared with those of PBS-treated mice (Fig. 3a). However, the levels of the three miRNAs in the serum markedly decreased from day 3 to day 5 after CTX injury (Fig. 3a). On the other hand, CK activity in serum of CTX-injured mice was about 2.0-fold higher than controls between day 1 and day 3 (Fig. 3b). Our data indicate that the levels of miR-1, miR-133a, and miR-206 in serum are strikingly up-regulated by muscle injury. 
Fig. 3 a Expression levels of miR-1, miR-133a, and miR-206 and $\mathbf{b} \mathbf{C K}$ activities in serum of mice on day 1,3 , and 5 after CTX $(n=3)$ injury or Mock $(n=3)$

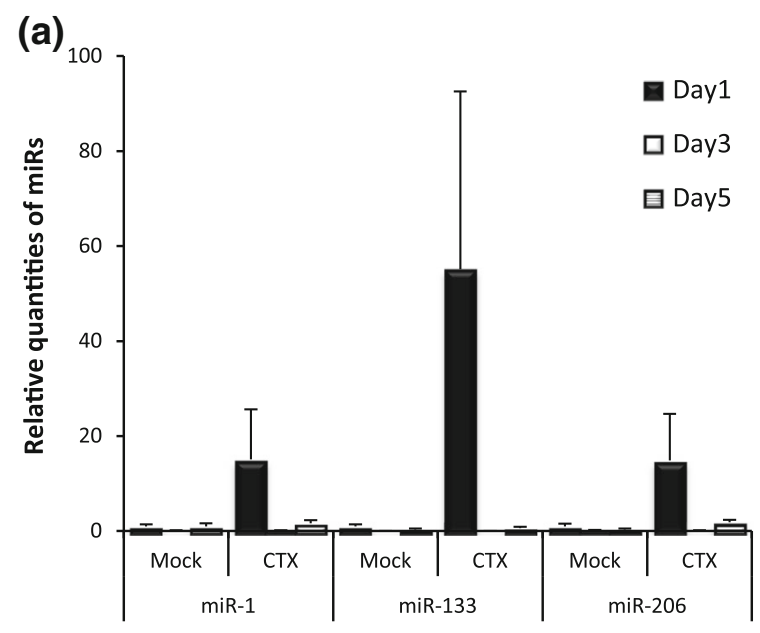

\section{Discussion}

In this study, miR-133a and miR-206 levels in exosome from the serum of DMD patients were increased compared to the exosome-depleted supernatant. However, it was reported that the miRNAs in $m d x$ mice were more enriched in the supernatant fraction rather than in the exosome, and associated with Argonaute-2 (Ago-2) and Apolipoprotein A-1 (ApoA-1) [21]. This discrepancy may be depended on some differences for a degree of severe and progressive degeneration of affected tissues between human and mouse. It was recently reported that the miR-1, miR-133a, and miR-206 are up-regulated in both exosomes and a skeletal muscle cell line, $\mathrm{C} 2 \mathrm{C} 12$ cells, and miRNA profiles in exosome alter during differentiation [22]. Furthermore, although the number of exosomes from $\mathrm{C} 2 \mathrm{C} 12$ myotubes released into extracellular compartment by Dexamethasone was not changed, the abundance of the miR-1 in exosome was increased [23]. These findings represent that the upregulations of miRNAs in serum of mice might be partly explained by their selective exports into exosomes induced by muscle degeneration and/or regeneration.

It was also reported that lack of miR-206 shows delay of muscle regeneration induced by CTX injury and more severe dystrophic phenotype in $m d x$ mice due to impair differentiation of SC [24]. In our study, these miR-1, miR133a, and miR-206 levels in the serum were up-regulated in response to CTX-induced injury. These suggested that excessive secretion of miRNAs might partly be a cause for muscle diseases.

In summary, we evaluated three miRNAs, miR-1, miR133a, and miR-206, as novel biomarkers for muscle disorders. Although all diseases examined in this study exhibited no statistically significant associations with these miRNA levels in serum by Bonferroni correction, associations of miR-1 levels with LGMD, FSHD, and miR-133a and miR-206 with BMD showed significant differences without corrections of multiple test. However, additional studies on increasing sample size are required to further confirm its usefulness as novel biomarker for muscle disorders.

Acknowledgments We thank Dr. Jun Tanihata for valuable discussions and critical reading of the manuscript.

Conflict of interests The authors report no conflict of interests.

\section{References}

1. Esteller M. Non-coding RNAs in human disease. Nat Rev Genet. 2011;12:861-74.

2. Eisenberg I, Eran A, Nishino I, Moggio M, Lamperti C, Amato AA, et al. Distinctive patterns of microRNA expression in primary muscular disorders. Proc Natl Acad Sci U S A. 2007;104:17016-21.

3. Eisenberg I, Alexander MS, Kunkel LM. miRNAS in normal and diseased skeletal muscle. J Cell Mol Med. 2009;13:2-11.

4. Valadi H, Ekström K, Bossios A, Sjöstrand M, Lee JJ, Lötvall JO. Exosome-mediated transfer of mRNAs and microRNAs is a novel mechanism of genetic exchange between cells. Nat Cell Biol. 2007;9:654-9.

5. Yao B, Li S, Chan EK. Function of GW182 and GW bodies in siRNA and miRNA pathways. Adv Exp Med Biol. 2013;768:71-96.

6. Mercuri E, Muntoni F. Muscular dystrophies. Lancet. 2013;381:845-60.

7. Emery AE. The muscular dystrophies. Lancet. 2002;359:687-95.

8. Takeshima Y, Yagi M, Okizuka Y, Awano H, Zhang Z, Yamauchi $Y$, et al. Mutation spectrum of the dystrophin gene in 442 Duchenne/Becker muscular dystrophy cases from one Japanese referral center. J Hum Genet. 2010;55:379-88.

9. Tsukamoto H, Inui K, Fukushima H, Nishigaki T, Taniike M, Tanaka J, et al. Molecular study of Duchenne and Becker muscular dystrophies in Japanese. $\mathrm{J}$ Inherit Metab Dis. 1991;14:819-24. 
10. Yamagata H, Miki T, Sakoda S, Yamanaka N, Davies J, Shelbourne P, et al. Detection of a premutation in Japanese myotonic dystrophy. Hum Mol Genet. 1994;3:819-20.

11. Udd B, Krahe R. The myotonic dystrophies: molecular, clinical, and therapeutic challenges. Lancet Neurol. 2012;11:891-905.

12. Mitsuhashi S, Kang PB. Update on the genetics of limb girdle muscular dystrophy. Semin Pediatr Neurol. 2012;19:211-8.

13. van Deutekom JC, Wijmenga C, van Tienhoven EA, Gruter AM, Hewitt JE, Padberg GW, et al. FSHD associated DNA rearrangements are due to deletions of integral copies of a $3.2 \mathrm{~kb}$ tandemly repeated unit. Hum Mol Genet. 1993;2:2037-42.

14. Ikeda-Sakai Y, Manabe Y, Fujii D, Kono S, Narai H, Omori N, et al. Novel mutations of the GNE gene in distal myopathy with rimmed vacuoles presenting with very slow progression. Case Rep Neurol. 2012;4:120-5.

15. Malm C, Sjödin TL, Sjöberg B, Lenkei R, Renström P, Lundberg IE, et al. Leukocytes, cytokines, growth factors and hormones in human skeletal muscle and blood after uphill or downhill running. J Physiol. 2004;556:983-1000.

16. Zatz M, Rapaport D, Vainzof M, Passos-Bueno MR, Bortolini ER, Pavanello Rde C, et al. Serum creatine-kinase (CK) and pyruvate-kinase (PK) activities in duchenne (DMD) as compared with becker (BMD) muscular dystrophy. J Neurol Sci. 1991;102:190-6.

17. Cacchiarelli D, Legnini I, Martone J, Cazzella V, D’Amico A, Bertini E, et al. miRNAs as serum biomarkers for Duchenne muscular dystrophy. EMBO Mol Med. 2011;3:258-65.

18. Mizuno H, Nakamura A, Aoki Y, Ito N, Kishi S, Yamamoto K, et al. Identification of muscle-specific microRNAs in serum of muscular dystrophy animal models: promising novel blood-based markers for muscular dystrophy. PLoS ONE. 2011;6:e18388.

19. Jia Y, Suzuki N, Yamamoto M, Gassmann M, Noguchi CT. Endogenous erythropoietin signaling facilitates skeletal muscle repair and recovery following pharmacologically induced damage. FASEB J. 2012;26:2847-58.

20. Couteaux R, Mira JC, d'Albis A. Regeneration of muscles after cardiotoxin injury I. Cytological aspects. Biol Cell. $1988 ; 62: 171-82$.

21. Roberts TC, Godfrey C, McClorey G, Vader P, Briggs D, Gardiner $\mathrm{C}$, et al. Extracellular microRNAs are dynamic nonvesicular biomarkers of muscle turnover. Nucleic Acid Res. 2013;41:9500-13.

22. Forterre A, Jalabert A, Chikh K, Pesenti S, Euthine V, Granjon A, et al. Myotube-derived exosomal miRNAs downregulate Sirtuin1 in myoblasts during muscle cell differentiation. Cell Cycle. 2014;13:78-89.

23. Hudson MB, Woodworth-Hobbs ME, Zheng B, Rahnert JA, Blount MA, Gooch JL, et al. miR-23a is decreased during muscle atrophy by a mechanism that includes calcineurin signaling and exosome-mediated export. Am J Physiol Cell Physiol. 2014;306:C551-8.

24. Liu N, Williams AH, Maxeiner JM, Bezprozvannaya S, Shelton JM, Richardson JA, et al. microRNA-206 promotes skeletal muscle regeneration and delays progression of Duchenne muscular dystrophy in mice. J Clin Invest. 2012;122:2054-65. 